GA-A22191

\title{
TRANSITION PHYSICS AND SCALING OVERVIEW
}

\author{
by \\ T.N. CARLSTROM
}

DECEMBER 1995 


\section{DISCLAIMER}

This report was prepared as an account of work sponsored by an agency of the United States Government. Neither the United States Government nor any agency thereof, nor any of their employees, makes any warranty, express or implied, or assumes any legal liability or responsibility for the accuracy, completeness, or usefulness of any information, apparatus, product, or process disclosed, or represents that its use would not infringe privately owned rights. Reference herein to any specific commercial product, process, or service by trade name, trademark, manufacturer, or otherwise, does not necessarily constitute or imply its endorsement, recommendation, or favoring by the United States Government or any agency thereof. The views and opinions of authors expressed herein do not necessarily state or reflect those of the United States Government or any agency thereof. 


\title{
TRANSITION PHYSICS AND SCALING OVERVIEW
}

\author{
by \\ T.N. CARLSTROM
}

This is a preprint of an invited paper presented at the Fifth Workshop on H-Mode Physics, September 18-20, 1995, Princeton, New Jersey, and to be printed in the Proceedings.

\author{
Work supported by \\ U.S. Department of Energy \\ Contract DE-AC03-89ER51114
}

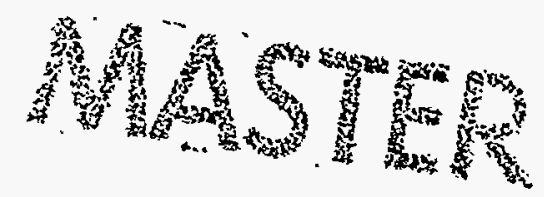

\section{GENERAL ATOMICS PROJECT 3466 DECEMBER 1995}




\title{
Transition physics and scaling overview
}

\author{
T.N. Carlstrom \\ General Atomics, San Diego, California, USA
}

\begin{abstract}
This paper presents an overview of recent experimental progress towards understanding H-mode transition physics and scaling. Terminology and techniques for studying $H$-mode are reviewed and discussed. The model of shear $E \times B$ flow stabilization of edge fluctuations at the $\mathrm{L}-\mathrm{H}$ transition is gaining wide acceptance and is further supported by observations of edge rotation on a number of new devices. Observations of poloidal asymmetries of edge fluctuations and dephasing of density and potential fluctuations after the transition pose interesting challenges for understanding $\mathrm{H}$-mode physics. Dedicated scans to determine the scaling of the power threshold have now been performed on many machines. A clear $B_{\mathrm{t}}$ dependence is universally observed but dependence on the line averaged density is complicated. Other dependencies are also reported. Studies of the effect of neutrals and error fields on the power threshold are under investigation. The ITER threshold database has matured and offers guidance to the power threshold scaling issues relevant to next-step devices.
\end{abstract}

Keywords. Fusion; tokamak; L to $\mathrm{H}$ transition; H-mode; radial electric field; shear stabilization; power threshold

\section{Introduction}

The H-mode continues to provide experimental and theoretical plasma physicists with a wealth of interesting and challenging opportunities to understand the complex behavior of magnetically confined plasmas. H-mode has now been observed in over 18 devices world wide, including most diverted tokamaks, stellarators, heliotrons, a tandem mirror, some limited tokamaks, and toroidal devices with biased electrodes. This portion of the workshop has benefited from the contribution of 20 papers on transition physics and scaling from 11 different institutions. The published proceedings of the last workshop may serve as a general reference on the prior progress of $\mathrm{H}$-mode physics (Plasma Phys. and Contr. Fusion 36 1994).

During its history of some 13 years (Wagner et al. 1982), the study of the H-mode transition has primarily focused on the understanding of the phenomena of the transition and the mechanisms by which the plasma spontaneously improves its particle and energy confinement. The exciting interplay between experiment and theory (reviewed in Groebner 1993, Burrell et al. $1994 \mathrm{~b})$ has led to a successful model of shear $E \times B$ flow stabilization of edge fluctuations at the $\mathrm{L}-\mathrm{H}$ transition which quenches anomalous turbulent transport and reduces particle and energy fluxes to low levels. However, fundamental questions on the origin of the radial electric field and the cause and effect relationship of turbulence suppression and electric field generation remain..

The transition to $\mathrm{H}$-mode is often observed to occur spontaneously whenever the input power to the plasma exceeds a threshold value. The plasma edge has been examined for a precursor or critical condition which acts as a trigger for the $\mathrm{L}-\mathrm{H}$ transition. The edge temperature, or something related to it, has been suspected for many years. The heat pulse associated with a sawtooth crash can often trigger an L-H transition and H-modes in only Ohmically heated plasmas are usually, if not always, triggered by sawteeth. The existence and/or relevance of a universally observed trigger for the $\mathrm{L}-\mathrm{H}$ transition remains an open question. 
More recently, the study of $\mathrm{H}$-mode has concentrated on the scaling of the power threshold so that access to this important confinement regime may be predicted for next generation machines such as ITER. The factor of two improvement in confinement obtained in $\mathrm{H}$-mode translates into large reductions in size and cost when extrapolating to reactor devices. An international database has now been established for this purpose (ITER H-mode database working group 1994, 1995).

A simple scaling relation that emerges from this database, which supports earlier investigations in this area (Carlstrom et al. 1989, Ryter et al. 1994), is that the power threshold scales like $n B S$, where $n$ is the line averaged density, $B$ is the toroidal field, and $S$ is the plasma surface area. Because of the high auxiliary power requirements this result has for ITER, it is being closely examined by experimental programs on several machines. A clear $B_{t}$ dependence is universally observed but dependence on the line averaged density is complicated. The existence of a low density threshold for the $\mathrm{L}-\mathrm{H}$ transition below which the power required to obtain H-mode increases is reported for several devices (Fielding et al. 1994, Greenwald et al. 1994, Anton et al. 1995, Miura et al. 1995). Studies of the effect of neutrals and error fields on the power threshold are also under investigation.

Recent studies of dimensionally similar scaling experiments on JET and DIII-D have shown the importance of the power threshold scaling on the design and performance of ITER (Luce and Petty 1995, Balet et al. 1995). They have examined a set of discharges dimensionally similar to ITER EDA 1994 except for the normalized gyro radius, $\rho_{*} \cdot \rho_{*}$ is one non-dimensional plasma parameter that can not be made equal to the ITER value in existing tokamaks. Therefore, the scaling of energy confinement with $\rho_{*}$ is important for predicting confinement properties in ITER. The parameter $\rho_{*}$ also has the largest effect on the cost of a machine because it depends on both size and $B_{\mathrm{t}}$. Their results show that, for powers well above the threshold, confinement has favorable gyro-Bohm like scaling. However, as the power level approaches the threshold, the confinement scaling leaves the gyro-Bohm scaling and follows the less favorable Goldstonlike power threshold scaling. This suggests that $\mathrm{H}$-mode transition physics may dominate the operational space of ITER because it is currently designed to operate near the power threshold. Dimensionless scaling studies such as this and physics studies based on energy confinement scaling relationships have all pointed to the need to determine accurately the power threshold and find ways to reduce it in reactor grade devices.

Besides acquiring predictive capabilities for $\mathrm{H}$-mode operation, the study of the scaling of $H$-mode may be a useful tool for understanding H-mode physics. The study of the conditions required to obtain $\mathrm{H}$-mode necessarily includes the study of $\mathrm{Ohmic}$ and $\mathrm{I}$-mode plasmas and their transport, especially near the plasma edge. The edge region presents challenges to the experimentalist because measurements are often hampered by poor diagnostic access, limited spatial resolution, and because techniques for measuring relevant parameters are not yet fully developed. The theorist also faces challenges of including a multitude of effects to models such as neutral penetration, impurities, SOL physics, flows, turbulence and electric fields.

The complicated state of affairs at the plasma edge has generated a need for experimentalists to provide local edge measurements of relevant parameters at high spatial and temporal resolution so that a physics understanding of the threshold power requirements can be made. Scaling of the conditions in L-mode that lead to $\mathrm{H}$-mode transitions with global parameters such as total current, magnetic field, line density, and total radiated power are too coarse to test transition theories in detail.

This paper is organized as follows: Section 2 reviews some of the common terminology in use and discusses several techniques that have been developed to study $\mathrm{H}$-mode physics and scaling. The next section reviews experimental progress in transition physics, followed by Section 4 on recent scaling results. Discussions, future plans, and conclusions are presented in the last section. 


\section{Terminology and technique}

Because of the large number of institutions and devices involved in the study of H-mode physics and scaling, it is important that the language be as accurate as possible so that meaningful intermachine comparisons can be made. In this section, several aspects of the terminology and experimental technique are reviewed in order to facilitate communication. Techniques for studying the formation of $E_{\mathrm{r}}$ and its role in $\mathrm{H}$-mode physics have been recently discussed (Burrell et al. 1994a, Moyer et al. 1995) and will not be repeated here.

\section{1. $H_{\alpha} / D_{\alpha}$ signals}

An almost universally accepted indicator of an $\mathrm{H}$-mode transition is the sudden drop in the $\dot{H}_{\alpha}$ signal due to the reduction of recycling when edge particle transport is reduced. Although this is not a sufficient condition to establish whether a discharge is in H-mode or not, it is often used as the indicator when the transition takes place. For many applications, this interpretation is adequate. However, examples exist when no such drop in the $H_{\alpha}$ intensity is observed, yet the plasma exhibits other characteristics of $\mathrm{H}$-mode such as the formation of an edge transport barrier evidenced by increased edge gradients of temperature and density and a reduction in fluctuation levels. These H-modes have been referred to as "transitionless $\mathrm{H}$-modes." Such observations have been reported for low density discharges in JT-60U (Mori et al. 1994) and have also been observed in DIII-D (Carlstrom et al. 1995a).

Depending on which plasma-wall interaction region the observer views, and the exact conditions of the plasma discharge, the $\mathrm{H}_{\alpha}$ signal may show various types of behavior at the $\mathrm{L}-\mathrm{H}$ transition, decreasing in some regions while increasing in others. Therefore, an observer must exercise caution when interpreting $\mathrm{H}_{\alpha}$ signals and using them as $\mathrm{H}$-mode signatures.

\subsection{Temporal and spatial scales}

$\mathrm{H}_{\alpha}$ signals are often used to determine the exact time and duration of the $\mathrm{L}-\mathrm{H}$ transition. As the temporal resolution of various diagnostics increases, the time scales of SOL transport and the recycling process must be included in any analysis which addresses cause and effect relationships based on $\mathrm{H}_{\alpha}$ signal timing.

When the duration of the transition is discussed, it is not always clear what phenomena is being addressed. It may be the response of the $\mathrm{H}_{\alpha}$ signal, the reduction in fluctuation levels, the development of a sheared $E \times B$ flow, or the increase in the pressure gradient. Detailed descriptions of the phenomena being reported are necessary for effective communication and. comparisons.

Several investigators have reported that the spatial extent of any rapid changes occurring at the transition such as poloidal rotation, density fluctuation suppression, and pressure gradient changes, are limited to about a poloidal ion gyro radius at the plasma edge. This is a very small region, amounting to about $1 \mathrm{~cm}$ or less for typical conditions on many present day tokamaks. This region may grow somewhat in time after the transition but the poloidal rotation and the steep gradient region are confined to the plasma edge.

\subsection{Dynamic behavior at the transition}

The dynamics of many observable parameters associated with the I-H transition exhibit a number of different behavior patterns. $\mathrm{H}_{\alpha}$ signals are often used to categorize these patterns which frequently vary according to the amount of awxiliary heating and its relation to the threshold power. Dithering is the name given to transitions that oscillate between $\mathrm{H}$-mode and L-mode and can be described as a limit cycle oscillation due to a difference in the response of the plasma to a rise in either $T$ or $n$ (Zohm et al. 1994). The early dithers are mostly I-mode with brief transitions to $\mathrm{H}$-mode, while the late dithers are mostly $\mathrm{H}$-mode with brief transitions 
to L-mode. The dithering phase of a transition can be very short and only contain one or two dithers. These dithers have been misleadingly called "transition ELMs" since they do not correspond to the same physics as ELMs.

$\mathrm{H}_{\alpha}$ signals can also show $\mathrm{L}$-mode fluctuations which may have similar appearance to small ELMs or dithers. However, the edge transport barrier, evidenced by increases in the edge temperature or density, is not observed (Zohm et al. 1995).

\subsection{Threshold power scaling techniques}

Attempts have been made to determine the power threshold from existence diagrams of conditions where $\mathrm{H}$-mode is observed and where it is not observed. These attempts are usually flawed because of the large hysteresis of the power required to maintain $\mathrm{H}$-mode and the rapid change of plasma parameters after the transition.

One of the difficulties in using a database to study the scaling of the power threshold is the large scatter in data which masks all but the strongest trends. Part of this problem is due to the nature of the transition and its sensitivity to many uncontrolled parameters. But part of the problem also lies with the experimental technique used to evaluate parameters which make up the database. Ideally, one would like to establish a steady Ohmic or L-mode plasma and then slowly increase the power until a transition is observed and record the value of the power just before the transition. The parametric dependence of the power threshold would be examined by changing the conditions of the steady Ohmic or L-mode plasma and repeating the power scan. One parameter would be investigated at a time until a multidimensional threshold space had been mapped out. Unfortunately, experimental time is usually at a premium and such detailed scans are not always possible. Often a plasma is not in steady state and something else is evolving as the power is scanned to search for the threshold. This may complicate the interpretation of the lata.

Parameter scans may also occur over time from one set of run days to another. Because of the sensitivity of the power threshold to machine cleanliness, impurity levels (Wagner et al. 1982), wall conditions (Carlstrom et al. 1994), and gas puffing levels (Wagner et al. 1985), these effects must be monitored. Machine conditions can vary substantially from one day to another and the experimenter will not be able to distinguish between a parametric dependence and a change in the overall machine behavior. One method of assessing the condition of a device is to establish a reference discharge which is repeated before and after a parameter scan is performed. The establishment of a reference discharge is essential for an accurate determination of the threshold power scaling over an extended period of time or changing machine conditions such as new wall conditioning techniques.

There is considerable evidence to suggest that the transition takes place at the edge of the plasma and therefore, local edge parameters should be important. Assuming that the edge parameters depend on the local heat flux, it is the power flow through the edge that is important. With this insight, we may more accurately determine the relevant power by defining the power threshold as

$$
P_{\mathrm{th}}=P_{\mathrm{ohm}}+P_{\mathrm{aux}}-d W_{\mathrm{p}} / d t-P_{\mathrm{rad}(\mathrm{core})},
$$

where $P_{\text {th }}$ is the threshold power, $P_{\text {ohm }}$ is the Ohmic input power given by

$$
P_{\text {ohm }}=V_{\text {surf }} \times I_{\mathrm{p}}-d W_{\mathrm{b}} / d t \text {, }
$$

$V_{\text {surf }}$ is the surface voltage, $I_{\mathrm{p}}$ is the plasma current, $d W_{\mathrm{b}} / d t$ is the time rate of change of the magnetic energy, $P_{\mathrm{aux}}$ is the auxiliary heating power, $d W_{\mathrm{p}} / d t$ is the time rate of change of the plasma energy, and $P_{\mathrm{rad}(\mathrm{core})}$ is the radiation loss from within the separatrix or the core plasma. When the threshold power is high, $P_{\text {aux }}$ is usually the dominant term and little error is made if the other terms are ignored. However, the other terms can be significant when the threshold power is low, such as in Ohmic H-mode, or when non-stationary discharges are evaluated. For example, using $V_{\text {loop }} \times I_{\mathrm{p}}$ to estimate $P_{\text {ohm }}$ can lead to large errors in some cases. $P_{\text {aux }}$ is intended 
to represent auxiliary power that is absorbed by the plasma and not the power delivered to the plasma. This presents additional difficulties for RF heating, where the power coupled to the plasma must be evaluated, and for NBI heating at low densities, where beam shine through can be significant.

. It may be argued that the total input power is more relevant for scaling to future machines than the power flow across the plasma edge. Indeed, the required auxiliary power must be based on core radiation losses and other power loss mechanisms. However, when developing a predictive scaling relation, it may be advantageous to separate the contributions from various effects since these effects may scale in different ways. This method may not only reduce the data scatter but also improve the physics understanding of the various effects.

Transitions with only Ohmic heating present several difficulties for determining the power threshold. The surface voltage is not always easy to determine. On DIII-D, a time dependent series of MHD equilibrium fits are used and the results fitted and smoothed. A second problem is that almost all Ohmic transitions are triggered by sawtooth crashes. The sawtooth provides a pulse of power to the plasma edge that is not easy to quantify. The intensity of the sawtooth pulse is a function of the plasma safety factor $q$, with sawteeth in low $q$ discharges significantly affecting the plasma edge. The effect of sawteeth on the flow of power to the plasma edge has not been quantified and a resolution to this problem has not yet been addressed.

Ohmic transitions have also created some difficulties with the language. H-mode originally referred to "high" confinement as opposed to "low" confinement observed when auxiliary heating was used. Calling the improved confinement regime "Ohmic $\mathrm{H}$-mode" in pure Ohmically heated plasmas made sense because it indicated "high" confinement relative to the Ohmic phase. The term "Ohmic L-mode" is now being used to distinguish between "Ohmic $\mathrm{H}$-mode" and standard Ohmic conditions. It is also used to describe cases where the Ohmic confinement and scaling are comparable to L-mode scaling relations such as ITER89-P (Yushmanov et al. 1990).

Power resolution is of concern especially for discharges with low threshold power. This is mostly a problem for NBI heating since RF and Ohmic power levels can be controlled more or less continuously. One method of varying the power of a fixed power NBI source is to modulate the source with a period less than the beam slowing down time. This method has been used on DIII-D with good results (Carlstrom et al. 1994). Stair step power scans are used to determine the threshold in a single plasma discharge. Power flow through the edge of the plasma is important, so the duration of each stair step should be a few energy confinement times in order to accurately reflect the steady state power. If a transition is observed soon after a power change, before an energy confinement time, then the power step is too large or local edge heating may be affecting the result. Since plasma current ramps are known to affect the transition (Toi et al. 1990), with positive ramps suppressing the transition and negative ramps enhancing it, it becomes problematic to survey Ohmic input power by ramping the plasma current.

Correlated parameters are almost always present and can significantly scatter and confuse the interpretation of the power threshold data. For instance, plasma-wall gaps and impurity production, density and neutral pressure, and $\mathrm{x}$-point location and shear or neutral pressure are usually correlated in some way. For a toroidal field scan of the power threshold, JT-60 has reported a correlation between density and toroidal field, so that the individual scalings are uncertain (Mori et al. 1994). Other correlations with density such as locked modes, run away electrons, and Marfe formation may influence the determination of the power threshold on density. Breaking these correlations is important for understanding the true dependencies of the power threshold.

At power levels at or near the threshold the plasma is often dithering or exhibits type III ELMs and confinement $B$ factors are often only slightly greater than one. There is concern that if ITER operates at the power threshold it may not attain the required $H$ factor of approximately two. Therefore, the important scaling for ITER is not just the power threshold for obtaining $\mathrm{H}$-mode but the power threshold for obtaining $\mathrm{H}$-mode with significant $\mathrm{H}$ factors. 


\section{Transition physics}

\section{1. $E_{\mathrm{r}}^{\prime}$ and $H$-mode}

The model of shear $E \times B$ flow stabilization of edge fluctuations and the associated reduction in transport continues to be the leading model for the improved confinement properties of II-mode (see, for example, Burrell 1994c, Itoh 1994, and references therein). This is true not only at the plasma edge where a transport barrier forms, but also in the core of the plasma where internal transport barriers form in VH, hot ion, and high beta modes.

This model has gained further support from a variety of experimental observations. The rapid increase of impurity poloidal rotation previously reported on DIII-D (Groebner et al. 1990), JFT-2M (Ida et al. 1990), and W7-AS (Wagner et al. 1994) has also been observed on COMPASS-D (Carolan et al. 1995). Large increases in the C III poloidal velocity are observed during ELM free periods. Through the radial force balance equation, this indicates a change in $E_{\text {r. }}$.

Indirect evidence of the formation of $E_{\mathrm{r}}$ in $\mathrm{H}$-mode comes from fast particle CX fluxes on ASDEX-U (Herrmann et al. 1995). Ion trajectory calculations, based on $E_{\mathrm{r}}$ profiles measured on DIII-D, show that for a given ion energy, ripple trapped fast ions are confined above a certain $E_{\mathrm{r}}$. More energetic ions require larger $E_{\mathrm{r}}$ for confinement and as the ions are confined, their $\mathrm{CX}$ flux increases. Fast ion fluxes are observed to increase just after an I-H transition. Increases in the higher energy fluxes are delayed, indicating that Er continues to increase after the transition.

A negative $E_{\mathrm{r}}$ is observed in the edge region of JET H-modes (Hawkes et al. 1995), but it develops slowly from pressure gradient terms in the radial force balance equation and no sudden change in the carbon impurity poloidal rotation associated with $E_{\mathrm{r}}$ at the transition is observed. This result implies that the formation of $E_{\mathrm{r}}$ is a result of the improved H-mode confinement and not the cause. At present, this conclusion is not supported by results from other devices. Further research is currently underway to investigate this result from the standpoint of diagnostic resolution and sensitivity and whether the large size of JET may influence the formation of $E_{\mathrm{r}}$.

Observations of the dynamics of the $E_{\mathrm{r}}$ formation on DIII-D indicate that, at the transition, the main contribution to the change in $E_{\mathbf{r}}$ comes from the primary ion poloidal rotation (Burrell et al. 1994, Moyer et al. 1995). However, within a short time, a few ms in some cases, the dominant contribution to $E_{\mathrm{r}}$ comes from the main ion pressure gradient term. [As pointed out at the last $\mathrm{H}$-mode workshop, there can be a difference in the magnitude and direction of the rotation between the main ions and impurity ions, but the inferred $E_{\mathrm{r}}$ is the same for both ions (Kim et al. 1994).] This suggests a two-stage process for the development of H-mode. A trigger to start the formation of $E_{r}$, which in the case of spontaneous transition in DIII-D, is associated with a change in the perpendicular rotation, followed by an increase in the edge pressure gradient which sustains and augments the $E_{\mathrm{r}}$ formation. Accordingly, the physics of the $\mathrm{L}-\mathrm{H}$ transition is linked with the physics of poloidal edge rotation.

Further support for the two phase process of $\mathrm{H}$-mode formation comes from comparisons of the effect of the grad B drift direction on the L-H and H-L transition in ASDEX-U (Ryter et al. 1995). While the power threshold for the $\mathrm{L}-\mathrm{H}$ transition differs by about a factor of two for the case where the grad $B$ drift is away from the $x$-point, the power at the $H-L$ transition is independent of the grad B drift direction. This indicates that the grad B drift effect is important in the initial formation of $\mathrm{H}$-mode but not in its sustainment.

Triggers to establish the seed $E_{\mathrm{r}}$ may come from other sources such as producing $E_{\mathrm{s}}$ directly through electrode biasing (Taylor et al. 1989, Weynants et al. 1991), or pellet injection on TUMAN-3 (Lebedev 1995) which may increase the pressure gradient term sufficiently.

In an alternative view, the seed $E_{\mathrm{r}}$ may be formed when electron particle transport across the plasma boundary is suddenly reduced due to the stabilization of an instability that causes turbulent particle transport. The interpretation of Langmuir probe measurements across the separatrix in CHS support this hypothesis (Toi et al. 1995). Once a seed $E_{\mathrm{r}}$ has been established, the plasma rapidly responds with poloidal rotation in order to maintain radial force balance. The 
sheared flows from this rotation further reduce transport and pressure gradients develop which reduce the rotation required by radial force balance. The increased pressure gradient also acts in a feedback loop, increasing $E_{\mathrm{r}}$ and further enhancing shear stabilization of the turbulence.

Large increases in the global particle confinement time are observed in CCT H-modes but energy confinement increases are insignificant, indicating that particle and energy transport is decoupled in the electrode-induced H-mode (Tynan et al. 1995). This may be an indication that different levels of $\mathrm{Er}$ shear may be needed to affect the particle flux and the heat flux.

The development and sustainment of a large negative $E_{\mathrm{r}}$ well at the plasma edge over a wide range of conditions has been documented for $\mathrm{H}$-mode plasmas in DIII-D (Gohil et al. 1995). The $E_{r}$ well is robust, occurs just inside the separatrix where the fluctuations are reduced, moves with the plasma edge, and is present throughout the entire $\mathrm{H}$-mode phase.

\section{2. $E_{\mathrm{r}}^{\prime}$ and fluctuations}

Details of edge fluctuations have been studied with reciprocating Langmuir probes in the edge of DIII-D (Moyer et al. 1995) and PBX-M (Tynan et al. 1994). Fluctuation driven transport is reduced in the edge region where the $E_{\mathrm{r}}$ well forms in H-mode. However, the complete picture is rather complicated. Density fluctuations are also reduced in the SOL where there is little change in $E_{r}^{\prime}$. This may be due to a decrease in the driving force as the density in this region also decreases. Potential fluctuations can increase in the $E_{\mathrm{r}}$ well region but they don't contribute to transport because they are out of phase with the density fluctuations. Late in the H-mode phase, fluctuation levels return to near their L-mode values but the dephasing of the potential and density fluctuations results in little transport.

Asymmetries in the spatial location of density fluctuations have been measured using various reflectometry techniques and FIR scattering on DIII-D (Doyle et al. 1992, Doyle et al. 1995), and reflectometry (Rhodes et al. 1993) and Langmuir probes on CCT (Ty,san et al. 1995). They find that reduction of fluctuations seen at the transition is localized to the low field side of the plasma and that there is little or no change in the fluctuation level on the high field side. This suggests that these fluctuations are ballooning in character and are consistent with the asymmetries in the divertor heat flux at the inner and outer strike points at the target plates. These asymmetries could also be due to variation in $E_{r}^{\prime}$ along the flux surface (Burrell et al. 1992, Rhodes et al. 1993). Another asymmetry worth noting is that H-modes have not been observed in plasmas which were limited on the outboard or low field side, while they are relatively common for plasmas limited in the inside and elsewhere.

\section{Scaling of the power threshold}

Because of the the need for accurate power threshold predictions in next generation devices, considerable research has been directed toward determining the scaling of the H-mode power threshold. To date, the bulk of the work has centered around the scaling with global plasma parameters such as $I_{\mathrm{p}}, B_{\mathrm{t}}$, the line averaged density, $n_{\mathrm{e}} \ell$, and the plasma configuration. Early surveys of the power threshold on DIII-D indicated a scaling with $n_{\mathrm{e}} \ell$ and $B_{\mathrm{t}}$ but a lack of scaling with $I_{\mathrm{p}}$ (Carlstrom et al. 1989, Burrell et al. 1989). This result was surprising since energy confinement time had just the opposite scaling with these parameters, that is no dependence on $n_{\mathrm{e}} \ell$ or $B_{\mathrm{t}}$ and a linear dependence on $I_{\mathrm{p}}$. Power threshold scaling consistent with this simple scaling has also been observed on ASDEX-U (Ryter 1993), JFT-2M, and JT-60U (Mori et al. 1994). Recent surveys of the power threshold on JET (Righi et al. 1995, Cordey et al. 1995), C-MOD (Greenwald et al. 1994, Snipes et al. 1995), and COMPASS (Fielding et al. 1994, 1995) have supported the $n_{\mathrm{e}} \ell * B_{\mathrm{t}}$ scaling over a certain range of operational space specific to each device. Although the exact dependence of the power threshold on $B_{\mathrm{t}}$ is not certain, there seems to be agreement that the threshold increases with $B_{\mathrm{t}}$. However, significant deviations from this scaling are apparent in individual scans or under particular circumstances. 
There has not been consensus, however, on these scaling results and the matter continues to be investigated. Recent surveys on JT-60U (Sato et al. 1995) have shown a $q$ scaling for $q>7$. The modulation of power flow to the edge plasma by a sawtooth crash and the different sawtooth behavior in individual machines may lead to the variations of the observed $q$ scalings. In addition, low $q$ operational limits vary in different machines and this may further confuse the interpretation of the $q$ scaling of the power threshold.

In addition to a power threshold, there is evidence for a low density threshold as well. Density thresholds have been reported on ASDEX (Wagner et al. 1982, 1985), DIII-D (Burrell et al. 1987), JFT-2M, JT-60U (Mori et al. 1994), ASDEX-U (Ryter et al. 1994), W7-AS (Wagner et al. 1995), C-MOD (Snipes et al. 1994, 1995), COMPASS-D (Fielding et al. 1994), and TCV (Anton et al. 1995).

The dependence on the density can be complex. Both the low and high density operational ranges for a given machine appear to have higher power thresholds. The high density range leads to the density scaling of the power threshold while the low density range leads to the density threshold. At modest densities, there may be no density dependence to the power threshold.

The reasons for density thresholds may be due to operational constraints for individual machines. In DIII-D the low density operational limit is set by the locked mode threshold. As this low density limit is approached, the power threshold for H-mode increases. However, when field error correcting coils are used, the locked mode density limit can be reduced, and at lower target density, H-mode transitions are observed at approximately the same power as in the moderate density cases. In the W7-AS stellarator, an interesting set of circumstances exist where $\mathrm{H}$-mode is obtained with ECH using one gyrotron but not with two gyrotrons. This is attributed to the decoupling of $T_{i}$ and $T_{e}$ at low density. When the density is raised, $T_{i}$ and $T_{e}$ are better coupled through improved $e-i$ collisionality and the H-mode remains with two gyrotrons. The threshold power also increases for low density ECRH H-modes in COMPASS-D where the density threshcld is observed to scale with $B_{\mathrm{t}}$ (Carolan et al. 1994). These risults suggest the variety of ways that the density can influence the operational range of a device and the plasma conditions required for $\mathrm{H}$-mode.

The effect of neutrals has been studied on JT-60U (Tsuchiya et al. 1995) where it is found that the required edge collisionality for the transition decreases with increasing edge neutral pressure, $n_{0 \text { edge. }}$ This is consistent with the observation that the use of heavy gas puffing to obtain high density leads to high neutral pressures and increased power thresholds. The role of neutrals may play an important role in $\mathrm{H}$-mode power threshold scaling and this is an active area of current research.

The $n_{\mathrm{e}} \ell * B_{\mathrm{t}}$ scaling of the power threshold has provided some organization of the ITER threshold database (ITER H-mode working group 1994). However, the database indicates that the parameter with the greatest leverage on the prediction of the threshold power for ITER is the plasma surface area $S$ or some dimension similar to $R^{2}$ ) and the use of this parameter has helped to normalize the threshold power of many devices covering a wide range of surface areas from $5.5 \mathrm{~m}^{2}$ in COMPASS-D to $145 \mathrm{~m}^{2}$ in JET. The surface area of ITER is about $1200 \mathrm{~m}^{2}$. Experiments to isolate the scaling of the power threshold with this parameter have been done for a small range of $S$ within a single machine on JT-60U (Sato et al. 1995) where a linear dependence was found. Preliminary experiments using ITER simulation discharges to make direct size comparison between DIII-D and JET, where $S$ varies by a factor of three, have begun (Carlstrom et al. 1995b).

The ITER threshold database has also provided a forum from which consensus on several aspects of threshold power scaling for diverted tokamaks has emerged. When the grad B drift direction is toward the $x$-point location, the power threshold is about half of what it is when the grad B drift is in the opposite direction. There is an isotope effect on the power threshold, with lowest powers occurring for deuterium and higher powers for hydrogen and helium. An interesting exception to this is the lack of an isotope effect in the W7-AS stellarator (Wagner et al. 1994). Divertors, low recycling, and wall conditioning all lower the power threshold. 
In an attempt to deal with the many problems of intermachine comparisons previously discussed, a selected subset of the ITER threshold database, chosen to reflect the best conditions for predicting the power threshold for ITER, has been analyzed (ITER H-mode working group 1995). Significant scatter in the data exist, both for individual machines and from intermachine comparisons, which is about an order of magnitude over most of the data range. The best agreement for the lowest power points between the devices (except for COMPASS-D) is obtained with a dimensionally correct scaling of $P_{\mathrm{th}} \sim n_{\mathrm{e}} \ell \times B_{\mathrm{T}} \times R^{2.5}$. However, due to the strong size scaling, this dependence gives the highest threshold power prediction for ITER of $150 \mathrm{MW}$ at a density of $0.5 \times 10^{20} \mathrm{~m}^{-3}$. Other scalings, which fit the lowest power points almost as well, predict power thresholds about half this amount. The data have also been analyzed using discriminant analysis and assuming a power law form. The result shows a much weaker size dependence of $R^{0.77}$, almost no density dependence, and an inverse dependence on elongation, $\kappa^{-0.51}$. However, these results do not seem to bear much resemblance to the published global scalings. Increasing the confidence level of these predictions will require better understanding of the data selection to more closely resemble the conditions expected in ITER and better controlled experiments in existing devices to isolate the important parameters.

Although the determination of the power threshold with global, engineering parameters such as $I_{p}, n_{e} \ell, B_{\mathrm{t}}$, and size are important for predicting $\mathrm{H}$-mode transitions in future devices, the evaluation of local edge parameters is essential for evaluating theories of the transition. Measurements of the edge plasma $(r / a=0.97)$ with ruby laser Thomson scattering on DIII-D (Carlstrom et al. 1989, Burrell et al. 1989) indicated that $T_{e} / B_{t}$ was approximately constant just before the transition. Limited data in the ITER threshold database from DIII-D and ECE measurements from JET are consistent with this result but significant scatter is present in the data. During ITER simulation experiments on JET (Righi et al. 1995), edge $T_{e}$ measured by LIDAR Thomson scattering are consistent with $T_{e} / B_{t}$ being a constant, suggesting the importance of the gyro radius (proportional to $T^{0.5} / B$ ). However, the spatial resolution of these measurements may not be sufficient to reflect the plasma conditions where the transition begins. Recent results using multipulse Thomson scattering and CER measurements on DIII-D indicate that $T_{e} \sim 50 \mathrm{eV}$ and $T_{\mathfrak{i}} \sim 140 \mathrm{eV}$ at the plasma edge $(r / a=0: 99)$ just before the transition over a wide range of parameters, including almost a factor of ten range in the edge electron density (Groebner et al. 1995). However, no dependence on $B_{\mathrm{T}}$ was observed. This data indicates the importance of the edge temperature and that the electron or ion collisionality alone cannot be the unique criterion for the transition. The difference in the edge temperature dependence among the surveys may be due to the different $r / a$ values where it is measured. Further work in this area is required so that detailed quantitative theory-experiment comparisons can be made.

Attaining normalized beta of approximately one has also been proposed as a condition for the transition, and data from COMPASS-D supports this hypothesis (Carolan et al. 1995).

\section{Summary}

Significant progress has been made toward unfolding the complex plasma behavior surrounding the $\mathrm{H}$-mode transition. The model of shear $E \times B$ flow stabilization of fluctuations at the transition has provided a framework from which we are able to explore the origin of $E_{\mathrm{r}}$ and the condition or trigger. for the transition. Evidence suggests a two-stage process may be involved in the formation of the edge $E_{r}$ and transport barrier. The behavior of fluctuations and their role in heat and particle transport remains a complex issue.

Surveys on many machines are contributing information on power thresholds. The scaling of the power threshold with global plasma parameters and the existence of a density threshold has not reached consensus. This may be due, in part, to the correlation of various plasma parameters and to the particular experimental technique employed. In particular, operational constraints may be contributing to the complex density dependence. However, a convincing scaling relation, given in its simplest form as $P_{\text {th }} \sim n B S$, is beginning to emerge from the ITER threshold database. Continued work in this area of refining techniques and quantifying 
additional relevant parameters, such as neutrals and error fields, will help increase the confidence level of the power threshold scaling. Better understanding of the underlying physics of the L-H transition through detailed quantitative comparisons between experiment and theory will help to isolate the required conditions for spontaneous $\mathrm{H}$-mode transitions. Edge temperature and toroidal field continue to be important parameters for the transition. Future challenges include connecting these quantities with the formation of the seed $E_{r}$ at the transition.

\section{Acknowledgments}

Useful discussions with Drs. K.H. Burrell, D.J. Campbell, R.J. Groebner, Y. Miura, and F. Ryter are gratefully acknowledged.

This is a report of work sponsored by the U.S. Department of Energy under Contract No. DE-AC03-89ER51114.

\section{References}

Anton M et al. 1995 Plasma Phys. and Contr. Fusion (this conference)

Balet B et al. 1995 in Proc. 22nd EPS Conf. on Controlled Fusion and Plasma Physics, (European Physical Society, Petit-Lancy, 1995) to be published.

Burrell K H et al. 1987 Phys. Rev. Lett. 591432

Burrell K H et al. 1989. Plasma Phys. and Contr. Fusion 311649

Burrell $\mathrm{K} \mathrm{H}$ et al. 1992 Plasma Phys. and Contr. Fusion 34615

Burrell K H et al. 1994a Phys. Plasmas 11536

Burrell K H et al. 1994b Plasma Phys. Contr. Nucl. Fusion Research, Vienna, (Vienna: International Atomic Energy Agency) to be published

Burrell K H et al. 1994c Plasma Phys. and Contr. Fusion 36291

Carlstrom T N et al. 1989 in Proc. 16th EPS Conf. on Controlled Fusion and Plasma Physics, v $13 b, p 241$

Carlstrom T N et al. 1994 Plasma Phys. and Contr. Fusion 36147

Carlstrom T N 1995a ITER expert meeting, San Diego, March

Carlstrom T N et al. 1995 Plasma Phys. and Contr. Fusion (this conference)

Carolan P G et al. 1994 Plasma Phys. Contr. Nucl. Fusion Research, Vienna, (Vienna: International Atomic Energy Agency) to be published

Carolan P G et al. 1995 in Proc. 22nd EPS Conf. on Controlled Fusion and Plasma Physics, (European Physical Society, Petit-Lancy, 1995) to be published.

Cordey J G et al. 1995 Plasma Phys. and Contr. Fusion (this conference)

Doyle E J et al. 1992 Plasma Phys. Contr. Nucl. Fusion Research, Vienna, (Vienna: International Atomic Energy Agency) to be published

Doyle E J et al. 1995 Plasma Phys. and Contr. Fusion (this conference)

Fielding S J et al. 1994 Plasma Phys. Contr. Nucl. Fusion Research, Vienna, (Vienna: International Atomic Energy Agency) to be published

Fielding S J et al. 1995 Plasma Phys. and Contr. Fusion (this conference)

Gohil et al. 1994 Nucl. Fusion 341057

Greenwald M et al. 1994 Plasma Phys. Contr. Nucl. Fusion Research, Vienna, (Vienna: International Atomic Energy Agency) to be published

Groebner R J et al. 1990 Phys. Rev. Lett. 643015

Groebner R J 1993 Phys. Fluids B 52343

Groebner R J et al. 1995 Plasma Phys. and Contr. Fusion (this conference)

Hawkes N C et al. 1995 in Proc. 22nd EPS Conf. on Controlled Fusion and Plasma Physics, (European Physical Society, Petit-Lancy, 1995) to be published.

Herrmann W et al. 1995 in Proc. 22nd EPS Conf. on Controlled Fusion and Plasma Physics, (European Physical Society, Petit-Lancy, 1995) to be published.

Ida K et al. 1990 Phys. Rev. Lett. 651364 
ITER H-mode database working group 1994 in Proc. 21st EPS Conf. on Controlled Fusion and Plasma Physics v $1, \mathrm{p} 334$

ITER H-mode database working group 1995 Plasma Phys. and Contr. Fusion (this conference) Itoh K 1994 Plasma Phys, and Contr. Fusion 36307

Kim J et al. 1994 Phys. Rev. Lett. 722199

Liebedev S V 1995 Plasma Phys. and Contr. Fusion (this conference)

Luce T C and Petty C C 1995 in Proc. 22nd EPS Conf. on Controlled Fusion and Plasma Physics, (European Physical Society, Petit-Lancy, 1995) to be published.

Moyer R A et al. 1995 Phys. Plasmas 22397

Miura $M$ et al. 1995 ITER expert meeting, San Diego, March

Mori $M$ et al. 1994 Plasma Phys. and Contr. Fusion 36 A39

Righi E et al. 1995 in Proc. 22nd EPS Conf. on Controlled Fusion and Plasma Physics, (European Physical Society, Petit-Lancy, 1995) to be published.

Rhodes T L et al. 1993 Nucl. Fusion 331787

Ryter F et al. 1994a Plasma Phys. and Contr. Fusion 36 A99

Ryter F et al. 1994b in Proc. 22nd EPS Conf. on Controlled Fusion and Plasma Physics, (European Physical Society, Petit-Lancy, 1995) to be published.

Sato $M$ et al. 1995 Plasma Phys. and Contr. Fusion (this conference)

Snipes J A et al. 1994 Nucl. Fusion 341039

Snipes J A et al. 1995 Plasma Phys. and Contr. Fusion (this conference)

Toi K et al. 1990 Phys. Rev. Lett. 641895

Toi K et al. 1995 Plasma Phys. and Contr. Fusion (this conference)

Tsuchiya K 1995 Plasma Phys. and Contr. Fusion (this conference)

Tynan G et al. 1994 Plasma Phys. and Contr. Fusion 36285

Tynan G et al. 1995 Plasma Phys. and Contr. Fusion (this conference)

Wagner F et al. 1982 Phys. Rev. Lett. 491408

Wagner F et al. 1985 Nucl. Fusion 251490

Wagner F et al. 1994 Plasma Phys. and Contr. Fusion 36 A61

Wagner $\mathrm{F}$ et al. 1995 Trans. Fusion Technol. 2732

Weynants $\mathrm{R} \mathrm{R}$ et al. 1991 in Plasma Physics and Controlled Nuclear Fusion, Washington (Vienna: International Atomic Energy Agency) v 1, p 473

Yushmanov P G et al. 1990 Nucl. Fusion 301999

Zohm $\mathrm{H}$ et al. 1995a Nucl. Fusion 35543

Zohm H et al. 1995b Plasma Phys. and Contr. Fusion 37437 
* general atomics

P. O. BOX 85608 SAN DIEGO, CA 92186-9784 (619) 455-3000 